\title{
Cognitive activity in limited time conditions: individual and typological features
}

\author{
Anna Talleva ${ }^{1, *}$, Natalia Zvyagina ${ }^{1}$ and Inga Zashikhina ${ }^{1}$ \\ ${ }^{1}$ Northern (Arctic) Federal University, Arkhangelsk, Russia
}

\begin{abstract}
Rapid socio-economic and technological innovations, quick pace of life, colossal information loads and limited time are highly common for the society of the XXI century. Reducing performance time is one of the powerful stress factors that affect the adaptation processes and the psychophysiological state of the body. The result of a temporary limitation in task implementation may result in the optimization of all processes in the body and maximized work efficiency or have an opposite effect. There are many physiological markers of stress response development in the human body, one of which is the parameters of eye movement. Evidence exists that representatives with different types of autonomic regulation of functions have specific manifestations of stress reactions that are traced in the oculomotor activity. In this research, seventy students of the Northern (Arctic) Federal University named after M.V. Lomonosov, Russia, were examined for the study of their oculomotor reactions. Students with different autonomic status solved cognitive tasks either in a limited or unlimited time. The most sensitive parameters of oculomotor reactions were revealed when participants were reading under a time limit. It has been discovered that representatives with different vegetative status have specific manifestations of cognitive activity under the conditions of restricted time. The study shows that the efficiency of processing visual information in a limited time is accompanied by an increase in frequency parameters and a decrease in the duration of fixations identified in representatives with normotonic vegetative status.
\end{abstract}

\section{Introduction}

The accelerated ways of the modern society, hectic lifestyles and demand of operating huge information loads within limited time make psychophysiological and psychoemotional balance somewhat problematic. Such cardinal changes require certain levels of physiological adaptation from an individual (Kahneman, 2006). In this situation, the time limit is a decisive factor influencing the adaptation processes and psychophysiological state of the body. Decreased time of work performance can optimize all processes in the body and lead to an increase in efficiency, and vice versa (Babaeva, Rotova \& Sabadosh, 2012; Bazarov \& Tumanyan, 2012).

There are many physiological markers of the body's response to stress agents, including limiting time factors. The parameters of these markers depend on the type of activity and the

\footnotetext{
*Corresponding author: a.taleeva@narfu.ru
} 
individual typological characteristics of the organism (Fokin, 2014). Numerous studies prove the fact of distinct individual differences in people's resistance to stress under conditions of psychoemotional stress (Sudakov, 2005). Koltsova comments that time limitation, as a stress factor, affects the organism of the examined patients in different ways, depending on their individual physiological and psychophysiological characteristics (Koltsova, 2003). There is evidence of the influence of personality traits on heart rate regulation while performing cognitive tasks under stress. There have been identified individual shifts in the functional state's indicators in a situation of mobilization and during cognitive tasks' performance when a maximum comfortable time of task performance in conditions of lack of time was insured. Krivolapchuk speaks of two main types of autonomic response to stress by children 12-13 years old. The first type is a response predominantly of a sympathetic type; the second is a response of a parasympathetic type (Krivolapchuk, Chernova \& Savushkina, 2015).

It is known that the stress response is accompanied by an increase in the work of the central and autonomic nervous systems, a boost of the blood supply to the brain, and changes in brain activity. A reflection of brain activity is the oculomotor activity. Oculomotor activity performs various functions - searching for information, scanning it, building and recognizing a visual image. The main components of eye tracking are saccades and fixations. Research by Merkulova and Kalinina confirms that in a stressful situation, there are changes in the parameters of oculomotor activity (Merkulova \& Kalinina, 2017). A sharp decline in the number of saccades also occurs during intense visual work, in particular, when determining visual acuity and recognizing small objects. However, in this case, smooth movements sharply increase in amplitude (Filin, 2002). At the time of a detailed object's examination, the duration of fixations on individual parts increases. The same data are consistent with the results of the study by Reyes and Lee, where changes in eye movement were documented during the use of the DriveSafety driving simulator (Reyes \& Lee, 2008). The studies by Hertzum and Holmegaard prove that performing a cognitive task under conditions of time constraints leads to changed pupil diameter and mental stress rise (Hertzum \& Holmegaard, 2013). Altered parameters of saccades, fixations, and pupil diameter may indicate the intensity of the cognitive visual tasks' performance, as well as interest in the visual object or its absence.

Thus, at present, studies are conducted on the influence of various stress factors on individual systems of the body. In a number of studies, a decrease in the time reserve is assessed as a stress factor. The effect of the stress factor on the body has not been practically studied, though. The carried out studies can hardly be considered complete and need more research. This fact determined the goal and objectives of our study.

\section{Methodology}

The research included 70 examined individuals, students of the Northern (Arctic) Federal University named after M.V. Lomonosov, Russia. The average age of the surveyed individuals was $19 \pm 1.5$ years. The study participants were somatically healthy, did not have acute or chronic diseases of the cardiovascular system, and did not have pathologies of the visual system. The study was conducted with the voluntary consent of the participants. The research was fulfilled outside the session period in the morning time and in compliance with the norms of biomedical ethics (Declaration of Helsinki and European Community directives, 8/609 EU). For the experiment, the students were invited in an isolated room where the effects of various distractions were minimized. The influence of the time limit on the efficiency of solving cognitive tasks, on the features of oculomotor activity and the functioning of the autonomic nervous system was determined. The listed research details allow us to evaluate the study as a comprehensive one. 
The features of visual-motor reactions based on tracking saccadic movements (eyetracking) were recorded using an iView $\mathrm{X}^{\mathrm{TM}} \mathrm{RED}$ device produced by the German company SMI (HSSMI). This equipment is designed for high-speed video recording of eye movements in the infrared range. The eye-tracking parameters were measured in a contact-free way with automatic compensation of head movement. In order to record the parameters of oculomotor activity, the subject was positioned in front of a 22 " monitor at a distance of $65-70 \mathrm{~cm}$ and an angle of $55-60^{\circ}$ from the system for remote registration of eye movements built into the monitor. Before recording eye tracking in the process of performing a cognitive task, calibration was carried out (recording was carried out with an error of no more than 0.1 ). After successful calibration, a fixation point was highlighted in the centre of the monitor screen; as the participant was ready to solve tasks, stimulus material was presented - words of 4 letters with a missing second letter, which had to be read aloud by inserting the missing letter. Reading the words was considered stimuli and cognitive load of the activity. Each stimulus contained eight lines of 5 words. The words were printed in 24 point size in Times New Roman font, black, the distance between adjacent words and lines was $1 \mathrm{~cm}$. All words were nouns in the nominative case in the singular. Reading a set of words was carried out in two-time modes: at an arbitrary pace and with a time limit. During the registration of oculomotor activity in the process of solving cognitive tasks, the correct answers and the time for completing the task were simultaneously recorded to calculate the effectiveness of cognitive activity and the processing time for one word. The average duration of one student's study was 2-3 minutes.

The obtained eye-tracking data were analyzed using the SMI BeGaze software package. For statistical analysis, the following main parameters of eye-tracking were chosen:

- saccade frequency,

- blinking frequency,

- average saccade duration,

- fixation frequency,

- average fixation duration,

- average spread,

- $\quad$ average amplitude.

To identify the vegetative status of the examined students, we used cardiointervalography recording in the first standard lead (left hand and right hand) using standard clamping electrodes (clothespins) and the VNS-spectrum hardware and software complex (Neurosoft, Ivanovo). The recording of the initial state, electrocardiogram indicators, was carried out in a state of calm wakefulness, in a sitting position, with normal, even breath. After recording the initial state, an orthostatic test was immediately performed according to the method of A.M. Wayne et al. The duration of each recording for the subject was 2 minutes (120 seconds). We recorded the temporal characteristics of the heart rate and some indicators of the variational pulsometry, according to Baevsky (2004).

Traditionally, the vegetative balance is determined by the value of IN in the state of calm wakefulness. However, changes in this indicator vary significantly in the orthostatic test even in individuals with the vagotonic type. In this regard, the vegetative status was determined not by the initial value of IN but by its dynamics (reactivity) in the orthostatic test. If the IN in the orthostatic test increased by more than $10 \%$ compared to the background value, such respondents were assigned to the group with the sympathotonic variant of reactivity (group 1). If the IN decreased, then such persons were assigned to the group with vagotonic reactivity (group 2). If the IN fluctuations remained within $\pm 10 \%$, such respondents were assigned to the group with the normotonic variant (group 3).

Statistical analysis of the studied parameters was carried out using a set of computer programs Statistical Package for the Social Sciences (SPSS) for Windows v.23.0. The method of nonparametric data processing based on the determination of medians $(\mathrm{Me})$ and 
quartiles (Q1-Q3) was chosen as a descriptive analysis. To study the structure of the relationships of the studied variables, correlation analysis with the calculation of Spearman's rank coefficient was used. The significance of differences was assessed using the Wilcoxon nonparametric signed-rank test. Nonparametric tests are accepted as most relevant because the distribution of the sample is not normal. In order to identify the significance of differences in the parameters of oculomotor activity between the groups, the Mann - Whitney test was used.

\section{Results and Discussion}

At the first stage of the study, the parameters of oculomotor activity were assessed with the examined groups solving cognitive tasks in various time conditions (in an arbitrary time mode and with a time limit). The most sensitive parameters of oculomotor activity during cognitive tasks' performance in different time conditions are:

- the frequency of fixations,

- the frequency of blinking,

- the average duration of fixation,

- the average spread (variance) of fixations.

It is for these parameters that significant differences were revealed when working in different time conditions.

The frequency of fixations per second in the process of reading limited in time increases significantly $(p=0.001)$ compared to similar parameters recorded at an arbitrary pace.

There were statistically significant differences in the frequency of blinks per second $(\mathrm{p}=$ 0.043). In free time mode, students blink more, as opposed to working under time constraints. Thus, in conditions of increased emotional stress (time limit), there is a greater concentration of attention on the task, and therefore there are fewer blinks.

The average duration of fixations was significantly longer in free-time mode $(p=0.001)$. This may indicate that working with a time limit students manage to process a more significant number of words while making a larger number of fixations of a shorter duration. That is, visual perception in the conditions of restricted time is characterized by an increase in the analytical component. At the same time, the dispersion of fixations decreases, which indicates the locality of visual perception and a deeper comprehension of the perceived information.

The quality of thought processes and other cognitive functions depends on the functional state of the organism. The study of individual parameters of the body's functional state was one of the stages of our research. To determine the vegetative status of students, the stress index was used. It is a marker of the psychoemotional state (Sobolnikov, 2019). Based on the obtained results, all subjects were divided according to the types of regulation of the autonomic nervous system (ANS): normotonics (20\%), sympathotonics (48\%), parasympathotonics $(32 \%)$.

Obviously, in subjects with different vegetative status, the realization of visual perception and thought processes in different time conditions will be accompanied by specific changes in the parameters of eye-tracking (Rendon-Velez, van Leeuwen, Happee, Horváth, van der Vegte, \& de Winter, 2016). Statistically significant differences in oculomotor reactions of representatives with different vegetative status were identified in terms of the following parameters: fixation frequency, frequency and delay of saccades.

The representatives with a sympathotonic vegetative status displayed significant differences in the oculomotor activity parameters in a free time mode and under conditions of a time limit in comparison with normotonic and parasympathotonic patients. In the free time mode, the frequency simpatotonics' fixations per second are significantly lower than that of normotonics $(p=0.025)$. Also, students with sympathotonic vegetative status revealed 
statistically significant differences in the parameters of fixation frequency, frequency and saccade delay under time-limited conditions. Representatives of this group had significantly lower parameters of the frequency of fixations and saccades and high parameters of saccades delay.

When comparing the studied parameters within the groups of the same vegetative status and taking into account different time conditions of work, we established statistically significant differences in the parameters of eye-tracking (Table 1). In the course of the analysis of the obtained data, it was revealed that the optimization of visual perception is implemented in different ways. The consequence of this is a different nature of changes in the parameters of eye tracking when working in an arbitrary mode and in conditions of a time limit in representatives with different vegetative status.

Table 1. Oculomotor activity parameters of students' solving cognitive tasks in different time conditions.

\begin{tabular}{|c|c|c|c|c|}
\hline Vegetative status & Indicator & \begin{tabular}{|c} 
Reading \\
during \\
unlimited time \\
Me (Q1; Q3)
\end{tabular} & $\begin{array}{l}\text { Timed reading } \\
\text { Me }(Q 1 ; \text { Q3) }\end{array}$ & $\begin{array}{c}\text { Asymptotic } \\
\text { significance. } \\
\text { p }\end{array}$ \\
\hline \multirow{8}{*}{ Normotonics } & $\begin{array}{l}\text { Fixation frequency (Ff). } \\
\text { pcs / s }\end{array}$ & $2.7(2.1 ; 3.3)$ & $3(2.3 ; 3.6)$ & $0.041^{*}$ \\
\hline & $\begin{array}{l}\text { Average duration of } \\
\text { fixations (DAf). ms }\end{array}$ & \begin{tabular}{|ll}
237.2 & $(206.7 ;$ \\
$340.2)$ & \\
\end{tabular} & $\begin{array}{|ll|}222.9 & (164.5 ; \\
301.1) & \\
\end{array}$ & $0.028 *$ \\
\hline & $\begin{array}{l}\text { Average spread (variance) } \\
\text { of fixations (DisAf). ms }\end{array}$ & $\begin{array}{|ll|}72.4 & (66.5 ; \\
81.9) & \\
\end{array}$ & $\begin{array}{|ll|}76.5 & (57.2 ; \\
126.5) & \\
\end{array}$ & 0.398 \\
\hline & $\begin{array}{l}\text { Saccades frequency (Fs). } \\
\text { pcs/s }\end{array}$ & $230(174 ; 284)$ & $162(130 ; 192)$ & 0.091 \\
\hline & $\begin{array}{l}\text { Average saccade duration } \\
\text { (DAs). ms }\end{array}$ & $2.8(2.3 ; 4.5)$ & $3.9(2.8 ; 4.8)$ & 0.176 \\
\hline & $\begin{array}{l}\text { Saccade amplitude (AAs). } \\
\text { deg }\end{array}$ & $\begin{array}{ll}36.8 & (34.5 ; \\
46.8) & \\
\end{array}$ & $46.5(37.2 ; 49.7)$ & 0.128 \\
\hline & $\begin{array}{l}\text { Saccade speed (VAs). deg/ } \\
\text { s }\end{array}$ & $3.3(3 ; 7.2)$ & $4.1(3.3 ; 7)$ & 0.236 \\
\hline & Saccade delay (Las). ms & $76.2(68 ; 113.9)$ & $\begin{array}{|ll|}82.7 & (59.7 ; \\
167.7) & \\
\end{array}$ & 1.0 \\
\hline \multirow{10}{*}{ Sympathotonics } & Blink rate (Bfr). pcs / s & $\begin{array}{ll}304.6 & \text { (184.4; } \\
401.3)\end{array}$ & $\begin{array}{|ll|}215.8 & (145.9 ; \\
301.6) & \\
\end{array}$ & 0.128 \\
\hline & $\begin{array}{l}\text { Fixation frequency (Ff). } \\
\text { pcs /s }\end{array}$ & $0.5(0.1 ; 1)$ & $0.5(0.1 ; 0.8)$ & 0.236 \\
\hline & $\begin{array}{l}\text { Fixation frequency (Ff). } \\
\text { pcs / s }\end{array}$ & $2.2(1.9 ; 2.5)$ & $2.4(2.1 ; 2.8)$ & $0.003 *$ \\
\hline & $\begin{array}{l}\text { Average duration of } \\
\text { fixations (DAf). ms }\end{array}$ & $\begin{array}{|lr|}296.3 & (226.7 ; \\
361.5) & \\
\end{array}$ & $\begin{array}{|lr|}264.3 & (203.7 ; \\
321.7) & \\
\end{array}$ & $0.004^{*}$ \\
\hline & $\begin{array}{l}\text { Average spread (variance) } \\
\text { of fixations (DisAf). ms }\end{array}$ & $\begin{array}{|lr|}78.4 & (59.7 ; \\
91.4) & \\
\end{array}$ & $67.8(57.8 ; 92.3)$ & $0.041^{*}$ \\
\hline & $\begin{array}{l}\text { Saccades frequency (Fs). } \\
\text { pcs/s }\end{array}$ & $2.2(2 ; 3.8)$ & $2.7(2.2 ; 2.9)$ & 0.334 \\
\hline & $\begin{array}{l}\text { Average saccade duration } \\
\text { (DAs). ms }\end{array}$ & $40.7(36.4 ; 42.4)$ & $39.2(37 ; 43.8)$ & 0.895 \\
\hline & $\begin{array}{l}\text { Saccade amplitude (AAs). } \\
\text { deg }\end{array}$ & $4(3 ; 5)$ & $3.9(3.6 ; 5.3)$ & 0.859 \\
\hline & $\begin{array}{l}\text { Saccade speed (VAs). deg } \\
\text { / s }\end{array}$ & $85(66.3 ; 109.6)$ & $84.5(73.7 ; 99.7)$ & 0.923 \\
\hline & Saccade delay (Las). ms & $\begin{array}{ll}409.6 & (222.1 ; \\
471.3)\end{array}$ & $\begin{array}{|ll|}333.4 & (308.3 ; \\
410.9) & \\
\end{array}$ & 0.337 \\
\hline Parasympathotonics & Blink rate (Bfr). pcs/s & $0.5(0.3 ; 0.8)$ & $0.4(0.2 ; 0.7)$ & 0.174 \\
\hline
\end{tabular}






Note: * values, the differences of which are significant at the level of $\mathrm{p} \leq 0.05$; Me is the median; (Q1 Q3) - quartiles.

Representatives of all three groups, solving cognitive tasks in different time conditions, demonstrated visual perception accompanied by changes in the static component: the frequency of fixations per second significantly increases, the average duration of fixations decreases $(p \leq 0.05)$ (Table 1). Parasympathotonics showed a decreases average duration of fixations at the tendency level. It should be noted that the frequency of fixations and the average duration of fixations have the same direction of changes in all three groups, depending on time conditions. The magnitude of these changes is, however, different.

Representatives with a sympathotonic vegetative status demonstrated significant differences

in the decrease of fixations' spread (dispersion) (at $p=0.041)$ (see Table 1). Changes in fixation variance indicate visual selectivity: lower fixation variance means smaller functional field of view, more localized image study, and more selective attention.

Analyzing the saccades' parameters of representatives with the same vegetative status but under different work time, significant differences in the magnitude of the amplitude and velocity of saccades were revealed. The latter was only true for the group with a parasympathetic vegetative status. When the time resource is limited, a significant increase in these parameters is observed (see Table 1). In this case, the dynamic oculomotor reactions' component of visual perception changes. It should be noted that under natural conditions, the duration of saccades is proportional to their amplitude and speed (with an increase in the saccade duration, the amplitude and speed also increase). We suppose that such a ratio of saccades' parameters is optimal and evolutionarily fixed. In limited time work conditions, representatives of this group increase the amplitude and speed of the saccade, while the saccade duration remains unchanged, which indicates that one saccade is scanning a larger field of visual information.

According to the results of statistical analysis, significant differences were found in representatives with the same vegetative status when comparing the parameters of solving cognitive tasks' efficiency and a word processing speed in different time conditions. Sympathotonics $(\mathrm{p} \leq 0.005)$ and parasympathotonics $(\mathrm{p}=0.018)$ revealed a significantly reduced efficiency of the cognitive task. Representatives with normotonic vegetative status showed increased efficiency of the cognitive task performance at the tendency level $(\mathrm{p}=$ 0.395). In all the three examined groups, word processing speed significantly decreases. Thus, representatives with normotonic vegetative status, performing cognitive tasks under constrained time conditions demonstrated changes in the functioning of the formed 
physiological system that provide the most effective and high-quality activity, which is also reflected in changes of the oculomotor activity parameters. Comparing the features of the statistical interaction of the studied parameters in representatives with different vegetative status, we can single out such correlations' changes that provide the most effective activity, taking into account the temporal conditions. (see Fig. 1).

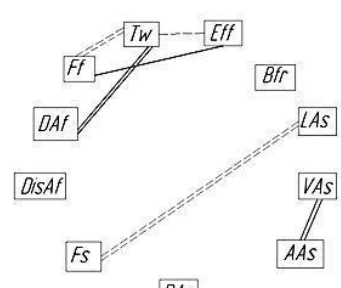

$D A S$



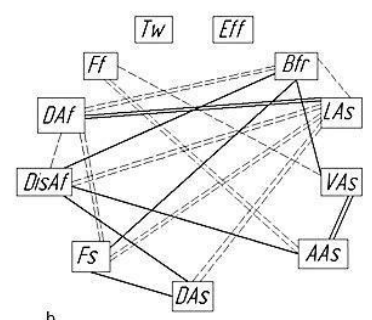



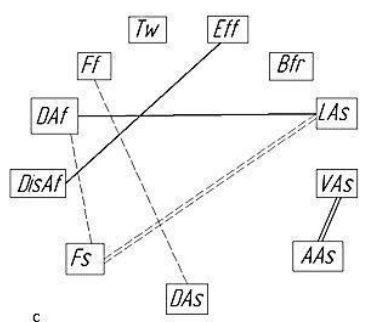

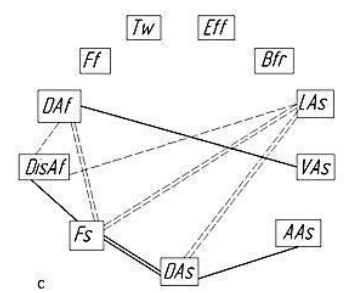

Fig. 1. Statistical interaction of the oculomotor activity parameters and the success of visual cognitive tasks' performance demonstrated by patients with different vegetative status.

Where:
a - normotonics
b - sympathotonics
c - parasympathotonics

The top row of correlation matrices denotes reading in free time mode;

The bottom row of correlation matrices denotes reading in limited time.

Ff - frequency of fixations,

DAf - average duration of fixations,

DisAf - average spread (variance) of fixations,

Fs - frequency of saccades,

DAs - average duration of saccade,

AAs - amplitude of saccade,

VAs - speed of saccade,

LAs - latent time (delay) of saccade,

Bfr - blinking frequency,

$\mathrm{Tw}$ - word processing time,

Eff - problem solution efficiency.

- direct correlations are statistically significant at the 0.05 level,

- - - inverse correlations, statistically significant at the 0.05 level

- direct correlations are statistically significant at the 0.01 level,

- - inverse correlations, statistically significant at the 0.01 level.

According to the assessment results of normotonics' activity effectiveness, it is the highest in comparison with the other participant groups. We conclude that these are the changes in the statistical interaction that are the most effective. In free time mode, representatives with a normotonic vegetative status reveal strong direct statistical 
relationships between the fixation parameters and the activity effectiveness, as well as the parameters of the saccades' speed and amplitude. In this case, strong relationships are formed between the saccades frequency and saccades latent time, and between fixations' frequency and word processing time per second, as well as between performance parameters and word processing time per second (see Fig. 1 (a)). That is, for normotonics, working in an arbitrary rhythm, the determining parameters of visual information perception are the saccades amplitude and speed and fixations duration and frequency. Activity effectiveness directly depends on the fixations' frequency; word processing time decreases with an increase in fixations' frequency and duration reduction. Performing a cognitive task under conditions of limited time, normotonic representatives have an increase in the direct interaction between their activity efficiency and fixation frequency. The temporal parameter of efficiency directly depends on the saccade delay and decreases with an increase in the saccade frequency.

The correlational array presents a variety of reliable forward and backward connections, the number and strength of which decreases with the introduction of a limiting time factor for representatives with a sympathotonic vegetative status reading during unlimited time (Fig. 1 (b)). Such changes in statistical interaction under the influence of a time limit are markers of the cognitive activity effectiveness fall.

The statistical interaction of the studied parameters for representatives with a parasympathotonic vegetative status performing the cognitive activity in an arbitrary time mode is minimized. The decisive factors for this activity are the speed and amplitudes of saccades, the latent time of saccades, as well as fixations parameters (see Fig. 1 (c). In this case, the activity effectiveness depends on the fixations' variance. Changes in the studied parameters' statistical interaction for this group's representatives, limited in time, exclude correlations with performance parameters.

\section{Conclusion}

Our studies have shown that visual perception of representatives with different vegetative status has specific features in different time conditions. It is known that the autonomic status of an organism, and hence the type of response to stress, is determined by the activation predominance of the sympathetic or parasympathetic ANS divisions. The ANS reaction type to unusual conditions is genetically determined, and therefore, the division of our study participants into groups according to the type of ANS response to stress is justified.

Acute lack of time is a powerful limiting factor that can trigger specific responses of the ANS, affect the efficiency and quality of work, energy and physiological costs. Undoubtedly, the time limit should be considered as an essential factor influencing the realization of cognitive functions.

Since the central and autonomic nervous systems' mechanisms and visceral functions are interconnected in the individual strategy of human adaptation to the limiting factors that create stressful conditions, the parameters of oculomotor reactions in the process of performing a cognitive task under conditions of time constraints can serve as markers of functional changes in the body and activity success. Our research has identified the most sensitive parameters of oculomotor activity under time-limited conditions - the fixations' frequency, the average fixations' duration, and the fixations' variance. It is critical that these markers can vary in representatives with different individual-typological characteristics of the vegetative status. Normotonics' sensitivity to the limiting time factor is mostly reflected by their parameters of fixations' frequency and average fixations' duration. Sympathotonics' demonstrate a higher fixations' frequency and average fixations' duration, as well as the variance of fixations. Parasympathotonics more often exhibit fixations' frequency and the amplitude and speed of saccades. Representatives of the identified groups in their process of cognitive work in different time conditions did not expose equal efficiency of task 
performance and word processing speed. Sympathotonics and parasympathotonics decreased their efficiency and task performance speed in restricted time mode. The severity of these changes is, however, different. Representatives with a normotonic vegetative status turned out to be the most efficient and speedy in their visual information processing under limited time conditions, which was ensured by an increase in their fixations' frequency and a decrease in their fixations' duration.

\section{Acknowledgements}

The research was carried out with the financial support of the RFBR in the framework of research project No. 19-313-90062.

\section{References}

1. M. Hertzum, K.D. Holmegaard, International Journal of Human-Computer Interaction 29(1), 26-39 (2013) doi.org/10.1080/10447318.2012.676538

2. V.V. Sobolnikov, Psychology of professional activity in special and extreme conditions: a textbook for universities (Yurayt Publishing House, Moscow, 2019)

3. K.V. Sudakov, Informational properties of functional systems and their mathematical modeling. Information models of functional systems (New Millennium Foundation, Moscow, 2004)

4. V.A. Filin, Automatic saccades (MSU Publishing House, Moscow, 2002)

5. V.F. Fokin, N.V. Ponomareva, V.I. Klopov, M.M. Tanashyan, O.V. Lagoda, Asymmetry 3, 4-22 (2014)

6. E. Rendon-Velez, P.M. van Leeuwen, R. Happee, I. Horváth, W.F. van der Vegte, J.C. F. de Winter, Transportation Research Part F: Traffic Psychology and Behavior 41, 150 169 (2016) doi.org/10.1016/j.trf.2016.06.013

7. M.L. Reyes, J.D. Lee, Transportation Research Part F: Traffic Psychology and Behavior 11(6), 391-402 (2008) doi.org/10.1016/j.trf.2008.03.004

8. Yu.D. Babaeva, N.A. Rotova, P.A. Sabadosh, Psychological research 5(25), 4 (2012) eISSN: 2075-7999

9. T.Yu. Bazarov, D.G. Tumanyan, National psychological journal 2(8), 116-123 (2012)

10. D. Kahneman, Attention and effort (Meaning, Moscow, 2006)

11. M.M. Koltsova, Slow Children (Speech, St. Petersburg, 2003)

12. I.A. Krivolapchuk, M.B. Chernova, E.V. Savushkina, New research 4(45), 24-32 (2015)

13. A.G. Merkulova, S.A. Kalinina, Hygiene and sanitation 96(8), 752-755 (2017) doi.org/10.1882/0016-9900-2017-96-8-752-755 